\title{
Constraints-preserving Transformation from XML Document Type Definition to Relational Schema ${ }^{\star}$
}

\author{
Dongwon Lee and Wesley W. Chu \\ Department of Computer Science \\ University of California, Los Angeles \\ Los Angeles, CA 90095, USA \\ \{dongwon, wwc $\}$ @cs .ucla.edu
}

\begin{abstract}
As Extensible Markup Language (XML) [5] is emerging as the data format of the internet era, there are increasing needs to efficiently store and query XML data. One way towards this goal is using relational database by transforming XML data into relational format. In this paper, we argue that existing transformation algorithms are not complete in the sense that they focus only on structural aspects and ignoring semantic aspects. We present the semantic knowledge that needs to be captured during the transformation to ensure a correct relational schema. Further, we show a simple algorithm that can 1) derive such semantic knowledge from the given XML Document Type Definition (DTD) and 2) preserve the knowledge by representing them in terms of semantic constraints in relational database terms. By combining the existing transformation algorithms and our constraints-preserving algorithm, one can transform XML DTD to relational schema where correct semantics and behaviors are guaranteed by the preserved constraints. Experimental results are also presented.
\end{abstract}

\section{Introduction}

As the World-Wide Web becomes a major means of disseminating and sharing information, Extensible Markup Language (XML) [5] is emerging as a possible candidate data format because it is simpler than SGML and more powerful than HTML. To query XML data, one way is to reuse the established relational database techniques by converting and storing XML data in relational storage. Since the hierarchical XML and the flat relational data models are not fully compliant, the transformation is not a straightforward task.

To this end, several XML-to-relational transformation algorithms have been studied $[8,9,16]$. Although they work well for the given applications, to a greater or lesser extent, they miss one important point. That is, the transformation algorithms only capture the structure of the Document Type Definition (DTD) and

* This research is supported in part by DARPA contract No. N66001-97-C-8601. 


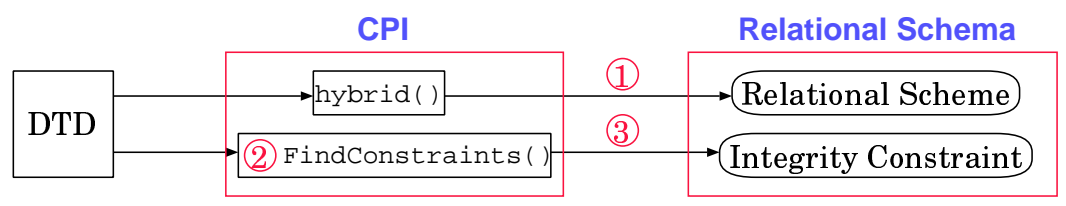

Fig. 1. Overview of our approach. Numbers 1) to 3) specify: 1) transforming schema, 2) discovering constraints via FindConstraints(), and 3) preserving constraints via RewriteConstraints().

ignore the semantic constraints hidden in it. In this paper, via our constraintspreserving inlining (CPI) algorithm, we show the kinds of semantic constraints that can be derived from DTD during transformation, and how to preserve them by re-writing them in resulting schema notation. Since our algorithm to capture and preserve semantic constraints from DTD is orthogonal to transformation algorithms, ours can be applied to various transformation algorithms in $[8,9,16]$ with little change. Figure 1 presents an overview of our approach. First, given a DTD, we transform it to a corresponding relational scheme using an existing algorithm. Second, during the transformation, we discover various semantic constraints in XML notation. Third, we re-write the discovered constraints to conform to relational notation.

This paper is organized as follows. Section 2 gives background information and related work. In Section 3, one transformation algorithm is discussed in detail. Section 4 presents various semantic constraints that are hidden in DTD. Section 5 proposes our algorithm to preserve such constraints during transformation. Section 6 reports some experimental results that we have conducted and Section 7 summarizes with concluding remarks.

\section{Background and Related Work}

Relational Schema: We define a relational schema $\mathcal{R}$ to be composed of a relational scheme $(\mathcal{S})$ and semantic constraints $(\Delta)$. That is, $\mathcal{R}=(\mathcal{S}, \Delta)$. In turn, the relational scheme $\mathcal{S}$ is a collection of table schemes such as $r\left(a_{1}, \ldots, a_{k}\right)$, where $a_{i}$ is the $\mathrm{i}$-th attribute in the table $r$ and the semantic constraints $\Delta$ is a collection of various semantic knowledge such as domain constraints, inclusion dependency, equality-generating dependency, tuple-generating dependency, etc.

XML and DTD: XML is a textual representation of the hierarchical data that is being defined by the World-Wide Web Consortium [5]. The meaningful piece of the XML document is bounded by matching starting and ending tags such as <name> and </name>. In XML, tags are defined by users while in HTML, permitted tags are pre-defined. Thus, XML is a meta-language that can be used for defining other customized languages. Using DTD, users can define the structure of the XML document of particular interest. A DTD in XML is very similar to a schema in a relational database. The main building blocks of DTD are elements 
Table 1. A DTD for Conference.

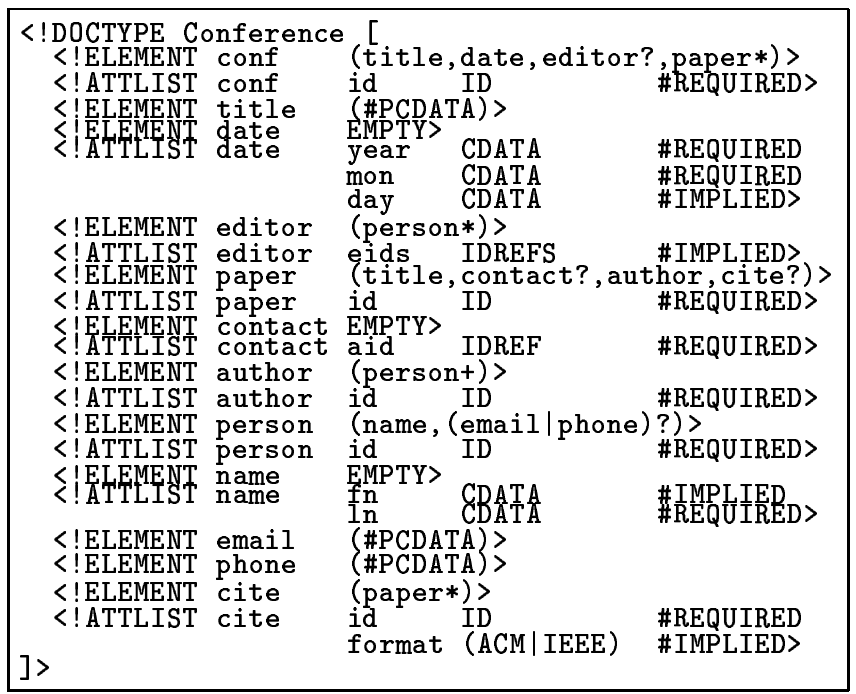

and attributes, which are defined by the keywords <! ELEMENT> and <! ATTLIST>, respectively. In general, components in DTD are specified by the following BNF syntax:

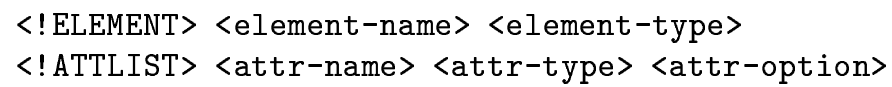

For a detailed description of DTD model, refer to [12]. Table 1 shows a DTD for Conference which states that a conf element can have four sub-elements: title, date, editor and paper in that order. As common in regular expression, 0 or 1 occurrence (i.e., optional) is represented by the symbol "?", 0 or more occurrences is represented by the symbol "*", and 1 or more occurrences is represented by the symbol "+". A sub-element without any such symbols (e.g., title) represents a mandatory one.

Keywords \#PCDATA and CDATA are used as string types for elements and attributes, respectively. For instance, the type of title element is defined as \#PCDATA so that title element can be arbitrary character data. <attr-option> can be \#REQUIRED or \#IMPLIED among others. An attribute with a \#REQUIRED option is a mandatory one while an attribute with a \#IMPLIED option is an optional one. <attr-type> keywords ID and IDREF are used for the pointed and pointing attributes, respectively. IDREFS is a plural form of IDREF. For instance, the author element must have a mandatory id attribute and this attribute is used when other attributes point to this attribute. On the other hand, the contact element has a mandatory aid attribute that must point to the id attribute of the contacting author of the current paper. One interesting definition in Table 1 is the cite element; it can have zero or more paper elements as sub-elements, thus 
Table 2. A valid XML document conforming to the DTD for Conference in Table 1.

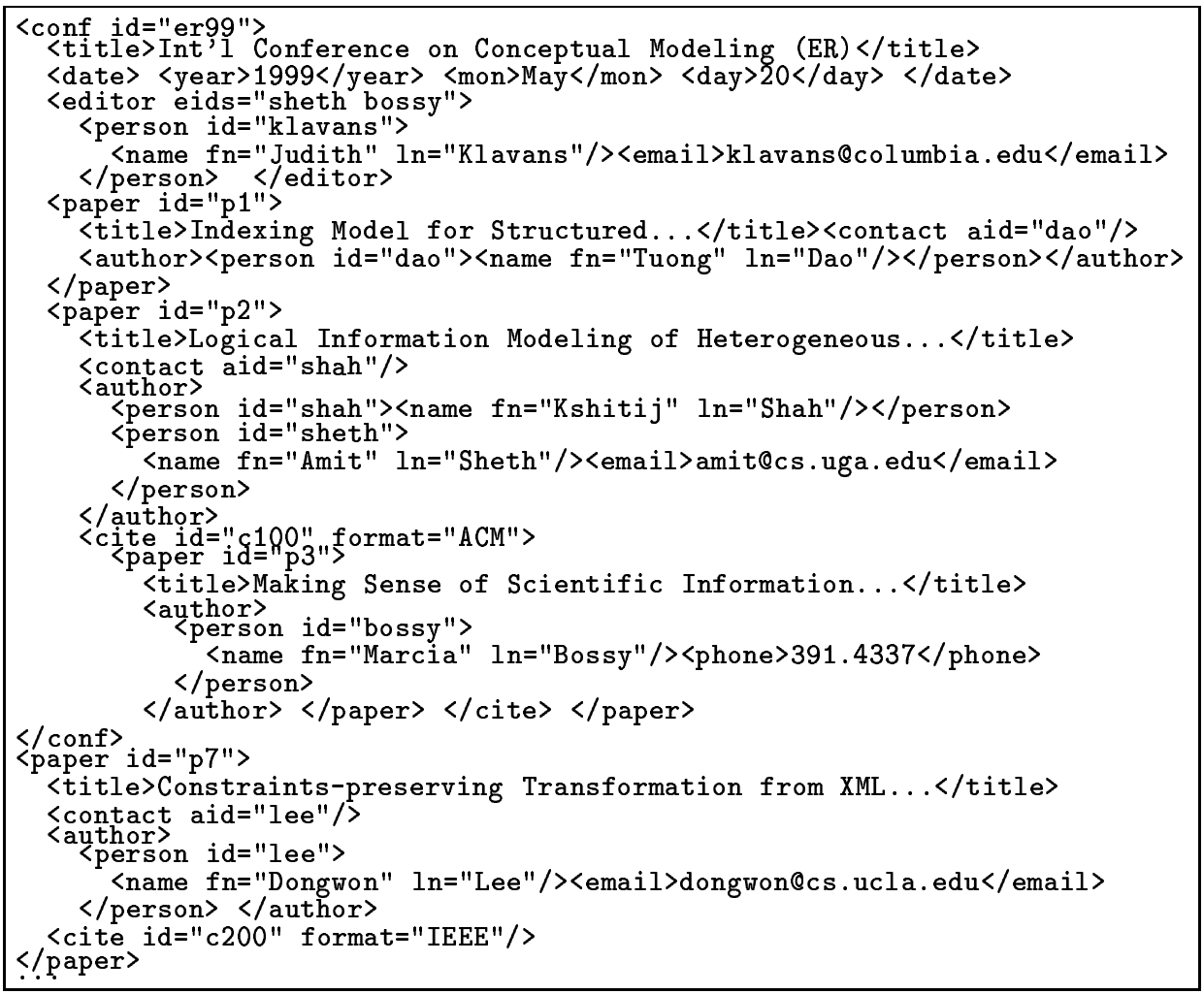

creating a cyclic definition. Table 2 shows a valid XML document conforming to the DTD for Conference. The document represents a portion of the fictional ER conference held in 1999. The first two paper elements are described with $i d=" p 1 "$ and $i d=" p 2 "$, respectively. The paper element with $i d=" p 2 "$ further has a cite element that describes the references in the paper. The paper element with id="p7" shows an example of the valid XML document that is not rooted at conf element. Note that a valid XML document can be rooted at any level of the DTD hierarchy as long as their sub-elements and attributes follow the DTD syntax.

Assumptions: Without loss of generality, to simplify our presentation, we assume that: 1) the input DTD has been already simplified using a technique in $[16], 2)$ the input XML documents are all valid, and 3) the XML features such as entities or notations are not covered.

Related Work: [16] presents three transformation algorithms that focus on the table level of the schema while [9] studies different performance issues among eight algorithms that focus on the attribute and value level of the schema. Since our CPI algorithm provides a systematic way of finding and preserving con- 
straints from a DTD, ours is an improvement to the existing transformation algorithms. Work done in STORED [8] deals with non-valid XML documents. When input XML documents do not conform to the given DTD, STORED uses a data mining technique to find a representative DTD whose support exceeds the pre-defined threshold. Since our algorithm to find and preserve constraints is not directly tied to a single transformation algorithm, ours can be applied to this algorithm as well. [13] also presents a DTD inference algorithm when it is not known. [4] discusses template language-based transformation from XML DTD to relational schema which requires human experts to write an XML-based transformation rule.

Some work has been done in [17] dealing with the transformation from relational tables to XML documents. There has been some transformation work in the OODB area as well [6]. Since OODB is a richer environment than RDB, their work is not readily applicable to our application. The logical database design methods and their associated transformation techniques to other data models have been extensively studied in ER research. For instance, [3] presents an overview of such techniques. However, due to the differences between ER and XML models, those transformation techniques need to be modified substantially.

\section{Transforming DTD to Relational Schema}

Transforming a hierarchical XML model to a flat relational model is not a trivial task. There are several difficulties including non 1-to-1 mapping, set values, recursion, and fragmentation issues [16]. For a better presentation, we chose one particular transformation algorithm, called the hybrid inlining algorithm [16] among many algorithms $[4,8,9,16]$. It is chosen since it exhibits the pros of the other two competing algorithms in [16] without severe side effects and it is a more generic algorithm than those in $[4,8]$. Since issues of discovering and preserving semantic constraints in this paper is orthogonal to that of transformation algorithms, our technique can be applied to other transformation algorithms easily.

\subsection{Hybrid Inlining Algorithm}

The hybrid algorithm [16] essentially does the following ${ }^{1}$ :

1. Create a DTD graph that represents the structure of a given DTD. A DTD graph can be constructed when parsing the given DTD. Its nodes are elements, attributes, or operators in DTD. Each element appears exactly once in the graph, while attributes and operators appear as many times as they appear in the DTD.

\footnotetext{
${ }^{1}$ We have made a few changes to the hybrid algorithm for a better presentation (e.g., renaming, supporting "|" operator), but the crux of the algorithm remains intact.
} 
2. Sub-elements in the choice model using the operator "|" are treated as if they are in the ordered sequence model with the following changes: 1) "+" operator is converted to "*" operator, 2) sub-elements without any occurrence operators are appended by "?" operator. For instance, <! ELEMENT A $((a \mid b)+\mid c)\rangle$ is converted to <! ELEMENT A $(a *, b *, c$ ? $)>$. Further, an attribute with \#IMPLIED or IDREFS type is converted to an operator node "?" or "+" in a DTD graph.

3. Identify top nodes in a DTD graph. A top node satisfies any of the following conditions: 1) not reachable from any nodes (e.g., source node), 2) direct child of " $*$ " or "+" operator node, 3) recursive node with indegree $>1$, or 4) one node between two mutually recursive nodes with indegree $=1$. Then, starting from a top node $T$, inline all the elements and attributes at leaf nodes reachable from $T$ unless they are other top nodes.

4. Attribute names are composed by the concatenated path from the top node to the leaf node using "_" as a delimiter. Use an attribute with ID type as a key if provided. Otherwise, add a system-generated integer key ${ }^{2}$.

5. If a table corresponds to the shared element with indegree $>1$ in DTD, then add a field parent_elm to denote the parent element to which the current tuple belongs. Further, for each shared element, a new field $\mathrm{fk} \_\mathrm{X} \$$ is added as a foreign key to record the key values of parent element $X$. If $X$ is inlined into another element $Y$, then record the $Y$ 's key value in the $f \mathrm{k}_{-} \$ \mathrm{Y} \$$ field.

6. Inlining an element $Y$ into a table $r$ corresponding to another element $X$ (i.e., top node) creates a problem when an XML document is rooted at the element $Y$. To facilitate queries on such elements, a new field root_elm is added to a table $r$.

7. If an ordered DTD model is used, a field ordinal is added to record position information of sub-elements in the element. (For simplification, the ordinal field is not shown in this paper.)

Table 3 shows the output of the transformation by the hybrid algorithm.

Among eleven elements in the DTD in Table 1, four elements - conf, paper, person, and eids - are top nodes and thus chosen to be mapped to the different tables. For the top node conf, the elements date, title, and editor are reachable and thus inlined. Then, the id attribute is used as a key and the root_elm field is added. For the top node paper, the elements title, contact_aid, author, cite_format and cite_id are reachable and inlined. Since the paper element is shared by the conf and cite elements (two incoming edges in a DTD graph), new fields parent_elm, $f k_{-}$conf and $f k_{-} c i t e$ are added to record who and where the parent node was. Note that in the paper table (Table 3), a tuple with id="p7" has the value "paper" for the root_elm field. This is because the element < paper $i d=" p 7 ">$ is rooted in the DTD (Table 2) without being embedded in other elements. Consequently, its parent_elm, $f k_{-}$conf and fk_cite fields are null. For the top node person, the elements name_fn, name_ln and email are reachable

\footnotetext{
${ }^{2}$ In practice, even if there is an attribute with ID type, one may decide to have a system-generated key for better performance.
} 
Table 3. A relational scheme $(\mathcal{S})$ along with the associated data that are converted from the DTD in Table 1 and XML document in Table 2 by the hybrid algorithm. Note that the hybrid algorithm does not generate semantic constraints $(\Delta)$.

\begin{tabular}{|c||c|c|c|c|c|}
\hline \multicolumn{5}{|c|}{ conf } \\
\hline id & root_elm & title & date_year & date_mon & date_day \\
\hline \hline er99 & conf & ER & 1999 & May & 20 \\
\hline
\end{tabular}

\begin{tabular}{|c||c|c|c|}
\hline \multicolumn{3}{|c|}{ conf_editor_eids } \\
\hline id & root_elm & fk_conf & eids \\
\hline \hline 100001 & conf & er99 & sheth \\
100002 & conf & er99 & bossy \\
\hline
\end{tabular}

\begin{tabular}{|c|c|c|c|c|c|c|c|c|}
\hline \multicolumn{9}{|c|}{ paper } \\
\hline id & root_elm & parent_elm & fk_conf & fk_cite & title & contact_aid & cite_id & cite_format \\
\hline p1 & 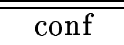 & $\overline{\text { conf }}$ & $\overline{\text { er99 }}$ & - & Indexing ... & $\overline{\text { dao }}$ & - & - \\
\hline $\mathrm{p} 2$ & conf & conf & er99 & - & Logical ... & shah & c100 & $\mathrm{ACM}$ \\
\hline p3 & conf & cite & - & c100 & Making ... & - & - & - \\
\hline p7 & paper & - & - & - & Constraints & lee & c200 & IEEE \\
\hline
\end{tabular}

\begin{tabular}{|c||c|c|c|c|c|c|c|c|}
\hline \multicolumn{10}{|c|}{ person } \\
\hline id & root_elm & parent_elm & fk_conf & fk_paper & name_fn & name_ln & email & phone \\
\hline \hline klavans & conf & editor & er99 & - & Judith & Klavans & klavans@cs... & - \\
dao & conf & paper & - & p1 & Tuong & Dao & - & - \\
shah & conf & paper & - & p2 & Kshitij & Shah & - & - \\
sheth & conf & paper & - & p2 & Amit & Sheth & amit@cs... & - \\
bossy & conf & paper & - & p3 & Marcia & Bossy & - & 391.4337 \\
lee & paper & paper & - & p7 & Dongwon & Lee & dongwon@cs... & - \\
\hline
\end{tabular}

and inlined. Since the person is shared by the author and editor elements, again, the parent_elm is added. Note that in the person table (Table 3), a tuple with id="klavans" has the value "editor", not "paper", for the parent_elm field. This implies that "klavans" is in fact an editor, not an author of the paper.

\section{Semantic Constraints in DTD}

Domain Constraints When the domain of the attributes is restricted to a certain specified set of values, it is called Domain Constraints. For instance, in the following DTD, the domain of the attributes gender and married are restricted.

$$
\begin{gathered}
<\text { !ATTLIST author gender (male|female) \#REQUIRED } \\
\text { married (yes } \mid \text { no) } \\
\text { \#IMPLIED> }
\end{gathered}
$$

In transforming such DTD into relational schema, we can enforce the domain constraints using SQL CHECK clause as follows:

CREATE DOMAIN gender VARCHAR(10) CHECK (VALUE IN ("male", "female")) CREATE DOMAIN married VARCHAR(10) CHECK (VALUE IN ("yes", "no")) 
When the mandatory attribute is defined by the \#REQUIRED keyword in DTD, it needs to be forced in the transformed relational schema as well. That is, the attribute In cannot be omitted below.

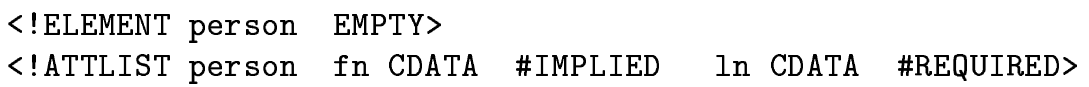

We use the notation " $X \not \nrightarrow \emptyset$ " to denote that an attribute $X$ cannot be null. This kind of domain constraint can be best expressed by using the NOT NULL clause in SQL as follows:

CREATE TABLE person (fn VARCHAR(20), In VARCHAR(20) NOT NULL)

Cardinality Constraints In DTD declaration, there are only 4 possible cardinality relationships between an element and its sub-elements as illustrated below:

<!ELEMENT article (title, author+, reference*, price?) >

A. 1-to- $\{0,1\}$ mapping ("at most" semantics): An element can have either zero or one sub-element. (e.g., sub-element price)

B. 1-to- $\{1\}$ mapping ("only" semantics): An element must have one and only one sub-element. (e.g., sub-element title)

C. 1-to- $\{0, \ldots\}$ mapping ("any" semantics): An element can have zero or more sub-elements. (e.g., sub-element reference)

D. 1 -to- $\{1, \ldots\}$ mapping ("at least" semantics): An element can have one or more sub-elements. (e.g., sub-element author)

For convenience, let us call each cardinality relationship as type A, B, C, and $\mathrm{D}$, respectively. From these cardinality relationships, mainly three constraints can be inferred. First, whether or not the sub-element can be null. Similar to the attribute case, we use the notation " $X \not \rightarrow \emptyset$ " to denote that an element $X$ cannot be null. This constraint is easily enforced by the NULL or NOT NULL clause. Second, whether or not more than one sub-elements can occur. This is also known as singleton constraint in [18] and is one kind of equality-generating dependencies. Third, given an element, whether or not its sub-element should occur. This is one kind of tuple-generating dependencies. The second and third types will be further discussed below.

Inclusion Dependencies (IDs) An Inclusion Dependency assures that values in the columns of one fragment must also appear as values in the columns of other fragments and is a generalization of the notion of referential integrity.

Trivial form of IDs found in DTD is that "given an element $X$ and its subelement $Y, Y$ must be included in $X$ (i.e., $Y \subseteq X$ )". For instance, from the conf element and its four sub-elements in DTD, the following IDs can be found as long as conf is not null: conf.title $\subseteq$ conf, conf.date $\subseteq$ conf, conf.editor 
$\subseteq$ conf, conf.paper $\subseteq$ conf $\}$. Another form of IDs can be found in the attribute definition part of DTD with the use of the IDREF(S) keyword. For instance, consider the contact and editor elements in the DTD in Table 1 shown below:

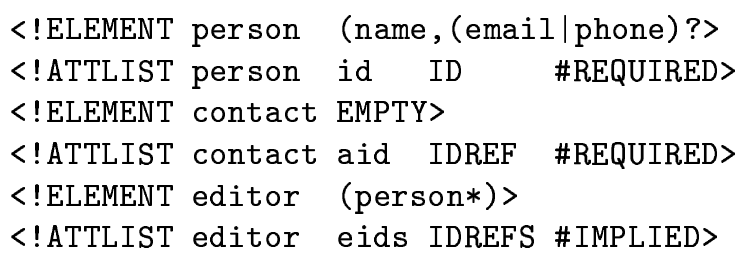

The DTD restricts the aid attribute of the contact element such that it can only point to the id attribute of the person element ${ }^{3}$. Further, the eids attribute can only point to multiple id attributes of the person element. As a result, the following IDs can be derived: editor.eids $\subseteq$ person.id, contact.aid $\subseteq$ person.id $\}$. IDs can be best enforced by the "foreign key" concept if the attribute being referenced is a primary key. Otherwise, it needs to use the CHECK, ASSERTION, or TRIGGERS facility in SQL.

Equality-Generating Dependencies (EGDs) The Singleton Constraint [18] restricts an element to have "at most" one sub-element. When an element type $X$ satisfies the singleton constraint towards its sub-element type $Y$, if an element instance $x$ of type $X$ has two sub-elements instances $y_{1}$ and $y_{2}$ of type $Y$, then $y_{1}$ and $y_{2}$ must be the same. This property is known as Equality-Generating Dependencies (EGDs) and denoted by " $X \rightarrow Y$ " in database theory. For instance, two EGDs: \{conf $\rightarrow$ conf.title, conf $\rightarrow$ conf.date\} can be derived from the conf element in Table 1. This kind of EGDs can be enforced by SQL UNIQUE construct. In general, EGDs occur in the case of the 1-to- $\{0,1\}$ and 1-to- $\{1\}$ mappings in the cardinality constraints.

Tuple-Generating Dependencies (TGDs) Tuple-Generating Dependencies $(T G D s)$ in relational model require that some tuples of a certain form be present in the table and use the " $\rightarrow$ " symbol. Two useful forms of TGDs from DTD are the child and parent constraints [18].

1. Child constraint: "Parent $\rightarrow$ Child" states that every element of type Parent must have at least one child element of type Child. This is the case of the 1-to- $\{1\}$ and 1 -to- $\{1, \ldots\}$ mappings in the cardinality constraints. For instance, from the DTD in Table 1, since the conf element must contain the title and date sub-elements, the child constraint conf $\rightarrow$ title, date $\}$ holds.

\footnotetext{
${ }^{3}$ Precisely, an attribute with IDREF type does not specify which element it should point to. This information is available only by human experts. However, new XML schema languages such as XML Schama and DSD can express where the reference actually points to [12].
} 


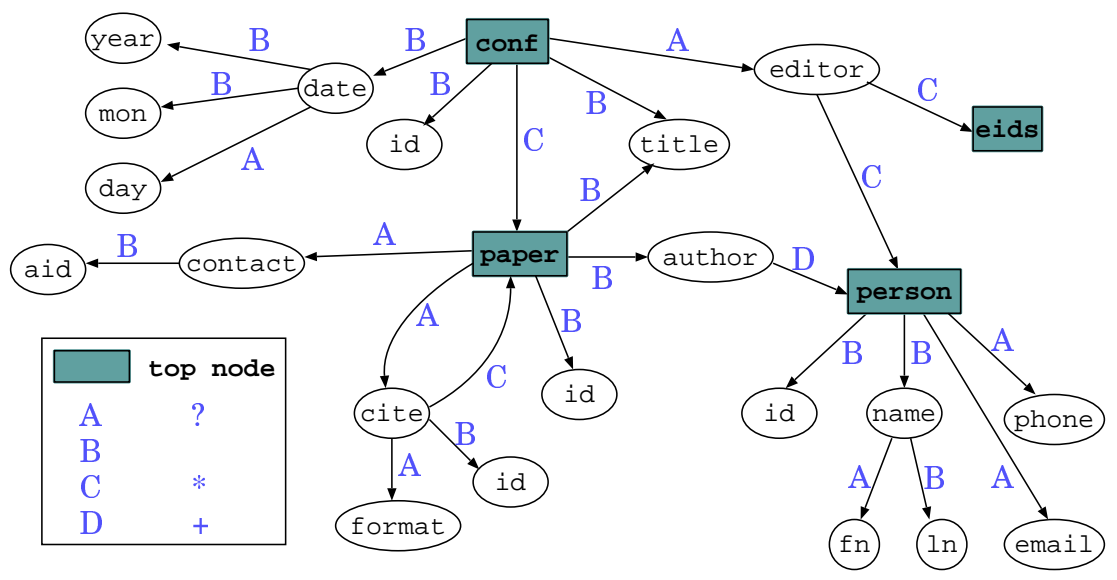

Fig. 2. An Annotated DTD graph for the Conference DTD in Table 1. The associated values of the nodes (i.e., indegree, type, tag, and status) are not shown.

2. Parent constraint: "Child $\rightarrow$ Parent" states that every element of type Child must have a parent element of type Parent. According to XML specification, there is no notion of root in DTD. That is, XML documents can start from any level of elements without necessarily specifying its parent element. Therefore, parent constraints cannot be assured simply by looking at the DTD structure. Rather, it requires some semantic knowledge. In the DTD in Table 1, for instance, the editor and date elements can have the conf element as their parent. Further, if we know that all XML documents were started at the conf element level rather than the editor or date level, then the parent constraint \{editor, date $\rightarrow$ conf holds. Note that the title $\rightarrow$ conf does not hold since the title element can be a sub-element of either the conf or paper element.

\section{Discovering and Preserving Semantic Constraints}

To help find semantic constraints, we use the following data structure:

Definition 1. An annotated DTD graph (ADG) $\mathcal{G}$ is a pair $(\mathcal{V}, \mathcal{E})$, where $\mathcal{V}$ is a finite set and $\mathcal{E}$ is a binary relation on $\mathcal{V}$. The set $\mathcal{V}$ consists of element and attributes in a DTD. Each edge $e \in \mathcal{E}$ is labeled with the cardinality relationship types ( $A$ to $D)$ as defined in Section 4. In addition, each vertex $v \in \mathcal{V}$ carries the following information:

1. indegree stores the number of incoming edges.

2. type contains the element type name in the content model of the DTD (e.g., conf or paper).

3. tag stores a flag value whether the node is an element or attribute (if attribute, it contains the attribute keyword like ID or IDREF, etc.). 
Table 4. Cardinality relationships and their corresponding semantic constraints.

\begin{tabular}{|r||c|c|c||c|c|c|}
\hline Relationship & Type & Symbol & Semantics & not null & EGDs & TGDs \\
\hline \hline 1 -to- $\{0,1\}$ & A & $?$ & at most & no & yes & no \\
$1-$ to- $\{1\}$ & B & & only & yes & yes & yes \\
$1-$ to- $\{0, \ldots\}$ & C & $*$ & any & no & no & no \\
$1-$ to- $\{1, \ldots\}$ & D & + & at least & yes & no & yes \\
\hline
\end{tabular}

4. status contains "visited" flag if the node was visited in a depth-first search or "not-visited".

Note that the cardinality relationship types in ADG considers not only element vs. sub-element relationships but also element vs. attribute relationships. For instance, from the DTD < !ATTLIST X Y \#IMPLIED Z \#REQUIRED >, two types of cardinality relationships (i.e., type A between element $X$ and attribute $Y$, and type B between element $X$ and attribute $Z$ ) can be derived. Figure 2 illustrates an example of ADG for the Conference DTD in Table 1. Then, the cardinality relationships can be used to find semantic constraints in a systematic fashion. Table 4 summarizes 3 main semantic constraints that can be derived from. The FindConstraints() algorithm can be immediately derived from the properties in Table 4. For detailed description, refer to [11].

Semantic constraints discovered by FindConstraints() have additional usage as we have shown in [11]. However, to enforce correct semantics in the newly generated relational schema, the semantic constraints in XML terms need to be re-written in relational terms. This is done by the algorithm RewriteConstraints().

\subsection{CPI: Constraints-preserving Inlining Algorithm}

We shall now describe our complete DTD-to-relational schema transformation algorithm: CPI (Constraints-preserving Inlining) algorithm is a combination of the hybrid inlining, FindConstraints() and RewriteConstraints() algorithms. The CPI algorithm is illustrated in CPI() and hybrid().

The algorithm first identifies all the top nodes from the ADG. This can be done using algorithms to find sinks or strongly-connected components in a graph [16]. Then, for each top node, the algorithm generates a corresponding table scheme using hybrid(). The associated constraints are found and re-written in relational terms using FindConstraints() and RewriteConstraints(), respectively. The hybrid() algorithm scans an ADG in a depth-first search manner while finding constraints and inlines a new field in the leaf node. The final output schema is the union of all the table schemes and semantic constraints.

Table 5 contains the semantic constraints that are re-written from XML terms to relational terms. As an example, the CPI algorithm will eventually spit out the following SQL CREATE statement for the paper table. Note that not only is the relational scheme provided, but the semantic constraints are also ensured by use of the NOT NULL, KEY, UNIQUE or CHECK constructs. 


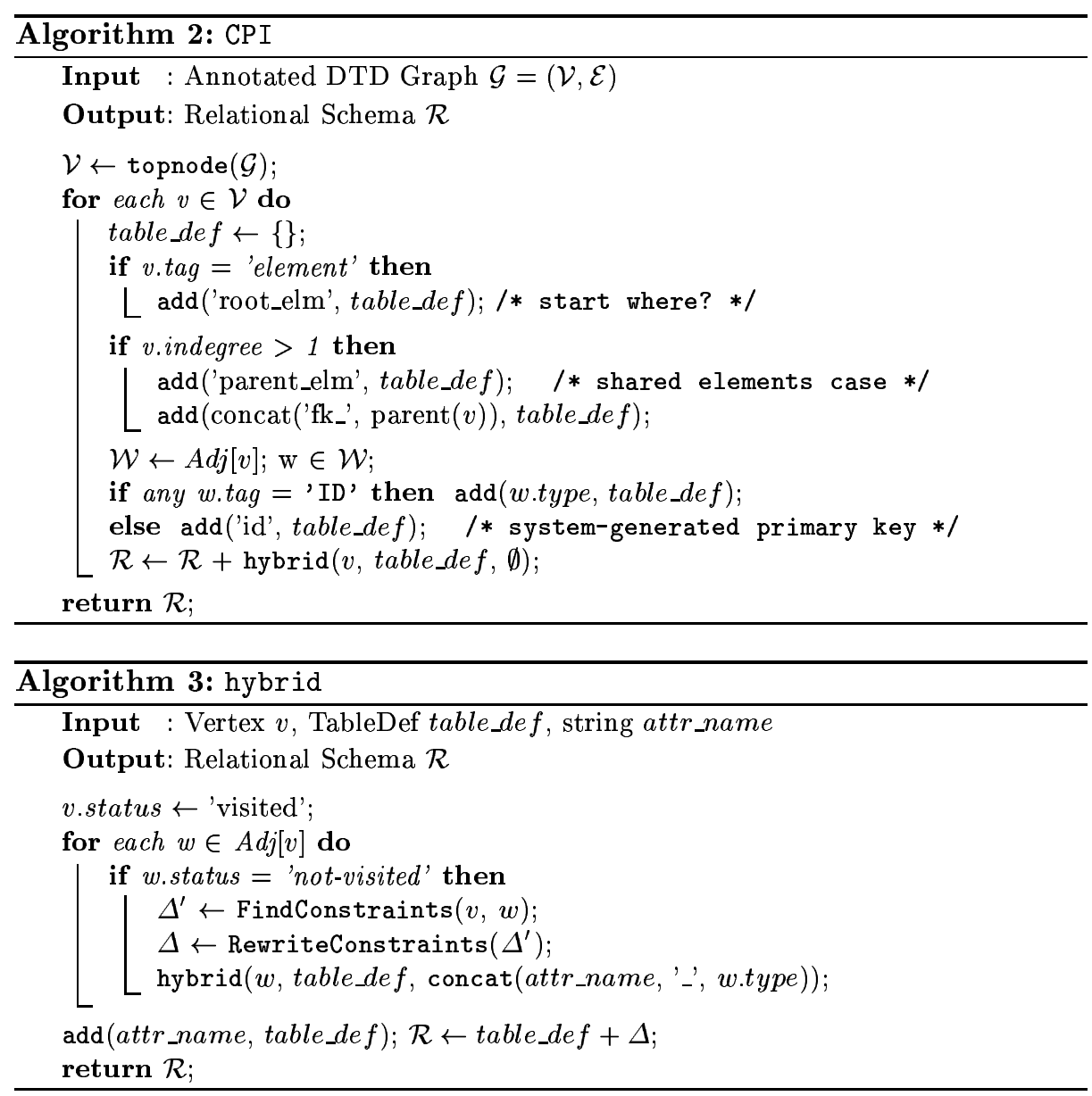

\section{Experimental Results}

We have implemented the CPI algorithm in Java using the IBM XML4J package. Table 6 shows a summary of our experimentation. We gathered test DTDs from "http://www.oasis-open.org/cover/xml.html" and [15]. Since some DTDs had syntactic errors caught by the XML4J, we had to modify them manually. Note that people seldom used the ID and IDREF(S) constructs in their DTDs except the XMI and BSML cases. The number of tables generated in the relational schema was usually smaller than that of elements/attributes in DTD due to the inlining effect. The only exception to this phenomenon was the XMI case, where extensive use of types $\mathrm{C}$ and $\mathrm{D}$ cardinality relationships resulted in many top nodes in the ADG.

The number of semantic constraints had a close relationship with the design of DTD hierarchy and the type of cardinality relationship used in the DTD. For instance, the XMI DTD had many type C cardinality relationships, which do 
Table 5. The semantic constraints in relational notation for the Conference DTD in Table 1.

\begin{tabular}{|c|c|}
\hline Type & Semantic constraints in relational notation \\
\hline$\overline{\mathrm{ID}}$ & conf_editor_eids[eids] $\subseteq$ person[id], paper[contact_aid] $\subseteq$ person[id] \\
\hline EGD & $\begin{array}{l}\text { conf[id] } \rightarrow \text { conf[title,date_year,date_mon,date_day] } \\
\text { paper[id] } \rightarrow \text { conf[title,contact_aid,cite_id,cite_format }] \\
\text { person[id] } \rightarrow \text { conf[name_fn,name_ln,email] }\end{array}$ \\
\hline TGD & $\begin{array}{l}\text { conf[id] } \rightarrow \text { conf[title,date_year,date_mon,date_day] } \\
\text { paper[id] } \rightarrow \text { conf[title,contact_aid,cite_id,cite_format] } \\
\text { person[id] } \rightarrow \text { conf[name_fn,name_ln,email], conf_editor_eids[fk_conf] } \subseteq \text { conf[id] } \\
\text { paper[fk_conf] } \subseteq \text { conf[id], paper[fk_cite] } \subseteq \text { paper[cite_id] } \\
\text { person[fk_conf] } \subseteq \text { conf[id], person[fk_paper] } \subseteq \text { paper[id] }\end{array}$ \\
\hline not null & $\begin{array}{l}\text { conf[id,title,date_year,date_mon,root_elm] } \nrightarrow \text { } \nrightarrow \\
\text { conf_editor_eids[id,root_elm] } \nrightarrow \emptyset \\
\text { paper[id,title,root_elm] } \nrightarrow \emptyset, \text { person[id,name_ln,root_elm }] \not \rightarrow \emptyset\end{array}$ \\
\hline
\end{tabular}

Table 6. Experimental results of the CPI algorithm.

\begin{tabular}{|c|c||c|c|c|c||c|c|c|c|c|}
\hline \multicolumn{2}{|c||}{ DTD } & \multicolumn{3}{c||}{ DTD Schema } & \multicolumn{3}{|c|}{ Relational Schema } \\
\hline Name & Domain & Elm & Attr & ID & IDREF(S) & Table & Attr & $\rightarrow$ & $\rightarrow$ & 只 $\emptyset$ \\
\hline \hline novel & literature & 10 & 1 & 1 & 0 & 5 & 13 & 6 & 9 & 9 \\
play & Shakespeare & 21 & 0 & 0 & 0 & 14 & 46 & 17 & 30 & 30 \\
tstmt & religious text & 28 & 0 & 0 & 0 & 17 & 52 & 17 & 22 & 22 \\
vCard & business card & 23 & 1 & 0 & 0 & 8 & 19 & 18 & 13 & 13 \\
ICE & content syndication & 47 & 157 & 0 & 0 & 27 & 283 & 43 & 60 & 60 \\
MusicML & music description & 12 & 17 & 0 & 0 & 8 & 34 & 9 & 12 & 12 \\
OSD & s/w description & 16 & 15 & 0 & 0 & 15 & 37 & 2 & 2 & 2 \\
PML & web portal & 46 & 293 & 0 & 0 & 41 & 355 & 29 & 36 & 36 \\
Xbel & bookmark & 9 & 13 & 3 & 1 & 9 & 36 & 9 & 1 & 1 \\
XMI & metadata & 94 & 633 & 31 & 102 & 129 & 3013 & 10 & 7 & 7 \\
BSML & DNA sequencing & 112 & 2495 & 84 & 97 & 104 & 2685 & 99 & 33 & 33 \\
\hline
\end{tabular}

not contribute to the semantic constraints. As a result, the number of semantic constraints at the end was small compared to that of elements/attributes in DTD. This was also true for the OSD case. On the other hand, in the ICE case, since it used many type B cardinality relationships, it resulted in many semantic constraints. For detailed discussions on the experimentation and the implementation of the CPI algorithm, please refer to [10].

\section{Conclusion}

This paper presents a method to transform XML DTD to relatonal schema both in structural and semantic aspects. After discussing the semantic constraints hidden in DTD, two algorithms are presented for: 1) discovering the semantic 
constraints using the hybrid inlining algorithm, and 2) re-writing the semantic constraints in relational notation. Our experimental results reveal that constraints can be systematically preserved during the conversion from XML to relational schema. Such constraints can also be used for semantic query optimization or semantic caching [11].

\section{References}

1. Abiteboul, S., Buneman, P., Suciu, D. "Data on the Web: From Relations to Semistructured Data and XML", Morgan Kaufmann Publishers, 2000.

2. Böhm, K., Aberer, K., Öszu, M. T., Gayer, K. "Query Optimization for Structured Documents Based on Knowledge on the Document Type Definition", Proc. IEEE Advances in Digital Libraries (ADL), Los Alamitos, California, April, 1998.

3. Batini, C., Ceri, S., Navathe, S. B. "Conceptual Database Design: An EntityRelationship Approach", The Benjamin/Cummings Pub. Inc., 1992.

4. Bourret, R. "XML and Databases", Internet Document, September, 1999. http://www.informatik.tu-darmstadt.de/DVS1/staff/bourret/xml/XMLAndDatabases.htm

5. Bray, T., Paoli, J., Sperberg-McQueen, C. M. (ed.), "Extensible Markup Language (XML) 1.0", W3C Recommendation, Feburary, 1998.

6. Christophides, V., Abiteboul, S., Cluet, S., Scholl, M. "From Structured Document to Novel Query Facilities", Proc. ACM SIGMOD, Minneapolis, Minnesota, 1994.

7. Deutsch, A., Fernandez, M. F., Florescu, D., Levy, A., Suciu, D. "XML-QL: A Query Language for XML", Proc. The Query Language Workshop (QL), 1998. http://www.w3.org/TR/NOTE-xml-ql

8. Deutsch, A., Fernandez, M. F., Suciu, D. "Storing Semistructured Data with STORED", Proc. ACM SIGMOD, Philadephia, Pennsylvania, June, 1998.

9. Florescu, D., Kossmann, D. "Storing and Querying XML Data Using an RDBMS", IEEE Data Engineering Bulletin, 22(3), September, 1999.

10. "XPRESS Home Page", 2000. http://www.cobase.cs.ucla.edu/projects/xpress/

11. Lee, D., Chu, W. W. "Constraints-preserving Transformation from XML Document Type Definition to Relational Schema (Extended Version)", UCLA-CS-TR 200001, 2000. http://www.cs.ucla.edu/ dongwon/paper/

12. Lee, D., Chu, W. W. "Comparative Analysis of Six XML Schema Languages", UCLA-CS-TR 200008, 2000. http://www.cs.ucla.edu/ dongwon/paper/

13. Ludäescher, B., Papakonstantinou, Y., Velikhov, P., Vianu, V. "View Definition and DTD Inference for XML", Proc. Post-ICDT Workshop on Query Processing for Semistructured Data and Non-Standard Data Formats, 1999.

14. Robie, J., Lapp, J., Schach, D. "XML Query Language (XQL)", WWW The Query Language Workshop (QL), December, 1998.

15. Sahuguet, A. "Everything You Ever Wanted to Know About DTDs, But Were Afraid to Ask", Proc. 3rd Int'l Workshop on the Web and Databases (WebDB), Dallas, TX, 2000.

16. Shanmugasundaram, J., Tufte, K., He, G., Zhang, C., DeWitt, D., Naughton, J. "Relational Databases for Querying XML Documents: Limitations and Opportunities", Proc. VLDB, Edinburgh, Scotland, 1999.

17. Turau, V. "Making Legacy Data Accessible for XML Applications", Internet Document, 1999. http://www.informatik.fh-wiesbaden.de/ turau/veroeff.html

18. Wood, P. T. "Optimizing Web Queries Using Document Type Definitions", Proc. 2nd Int'l Workshop on Web Information and Data Management (WIDM), 1999. 